\title{
X-ray Photoelectron Diffraction: Probing Atom Positions and Molecular Orientation at Surfaces
}

\author{
Roman Fase ${ }^{a \star}$ and Philipp Aebi ${ }^{\mathrm{b}}$
}

\begin{abstract}
With the growing need to gain control over atomic scale objects in nanosciences new tools have to be developed. Quantitative structural information is fundamental to the understanding of the physical properties, e.g. for the interpretation of spectroscopic results or as a starting point for theoretical calculations. This review will focus on the angle-resolved photoemission experiment to probe the geometrical structure on the atomic scale. Using soft $X$-rays shallow core levels can be excited for a chemical analysis of the surface. Photoelectrons emitted from a particular atom are mapped as a function of emission angle in the so-called photoelectron diffraction experiment. From the interference between the photoelectron wave directly reaching the detector and the waves scattered from the neighboring atoms we obtain very direct knowledge of the local real-space environment of the emitting atom. Photoelectron diffraction is able to determine atom positions at surfaces, to distinguish between different crystallographic surface structures, to identify impurity or dopant site positions or to unravel molecular orientation.
\end{abstract}

Keywords: Atomic-scale structure $\cdot \mathrm{C}_{60} \cdot$ Molecular orientation $\cdot$ Photoelectron diffraction

\section{Introduction}

With the current course of development in nanoscience and technology there is increasing need to characterize surfaces and artificial structures on ever smaller scales, ultimately reaching the atomic scale. For systems such as metallic clusters, semiconductor nanostructures, or supramolecular systems, quantitative structural information is fundamental to the understanding of their physical properties. Such nanostructures are usually organized at surfaces and surface science techniques are needed to characterize chemical composition and structure. Photoemission has a long-standing tradition in surface analysis. X-ray photoelectron spectroscopy (XPS) is used to study the chemical composition of surfaces. On single crystals, angle-resolved photoemission is able to study the atomic struc-

\footnotetext{
${ }^{\star}$ Correspondence: Dr. R. Fasela

aEMPA Dübendorf

Überlandstrasse 129

$\mathrm{CH}-8600$ Dübendorf

Tel.: +4118234348

Fax: +4118234034

E-Mail: Roman.Fasel@empa.ch

bInstitut de Physique

Rue A.-L. Breguet 1

CH-2000 Neuchâtel
}

ture, i.e. atomic positions, with a technique called X-ray photoelectron diffraction (XPD). Traditionally photoemission experiments have been performed by integrating over a large solid angle in order to maximize signal, or in angle-resolved form for rather few angles in order to vary the surface sensitivity of the experiment based on the finite escape depth of emitted electrons. Here we discuss experiments relying on extensive angle-scanning, covering much of the hemisphere above the sample surface as illustrated in Fig. 1a.

The aim of this review is to give an introduction and an illustration of results obtained by means of photoelectron diffraction. These examples will show that XPD can be used in different ways, $e$.g. to determine atom positions at surfaces, to distinguish between different crystallographic surface structures, as a simple finger-printing technique to identify impurity or dopant site positions or to unravel molecular orientation. In section 2 an introduction to anglescanned XPD is presented with emphasis on the so-called 'forward-focusing' regime. Different examples are presented in section 3 with an emphasis on the molecular orientation of $\mathrm{C}_{60}$ and $\mathrm{C}_{70}$ deposited on different substrates. Conclusions are given in section 4.

\section{Experimental Method}

Fig. 1 presents the general idea of the experiment. Two types of photon sources are available, one in the soft X-ray regime and the second in the ultraviolet (UV) range of energies. They are fixed in space and consist, in the present case, of a dual $\mathrm{MgK}_{\alpha}$ $/ \mathrm{SiK}_{\alpha} \mathrm{X}$-ray anode $(\mathrm{hv}=1254 \mathrm{eV} / 1740 \mathrm{eV})$ and a He lamp (HeI (21.2 eV)/HeII (40.8 $\mathrm{eV})$ ). Of course, synchrotron radiation, covering a wide energy range, is the ideal excitation source. In the present review we will not discuss excitation using UV radiation, which is important for valence band studies of the electronic structure. The electrostatic, hemispherical, angle and energy resolving analyzer is also fixed in space, and angle-scanning is performed by motorized sequential sample rotation [1]. Typically 4000 to 6000 angular settings are scanned, homogeneously distributed over the hemisphere above the sample.

The basis of XPD is the photoelectric effect. As photons of fixed energy are absorbed via the photoelectric effect, photoelectrons are emitted at different kinetic energies $E_{\text {kin }}$ according to $E_{\text {kin }}=-\left|E_{B}\right|+h v$, with $E_{B}$ the binding energy and hv the photon energy. The situation is sketched in Fig. $1 \mathrm{~b}$ where two different atomic species 


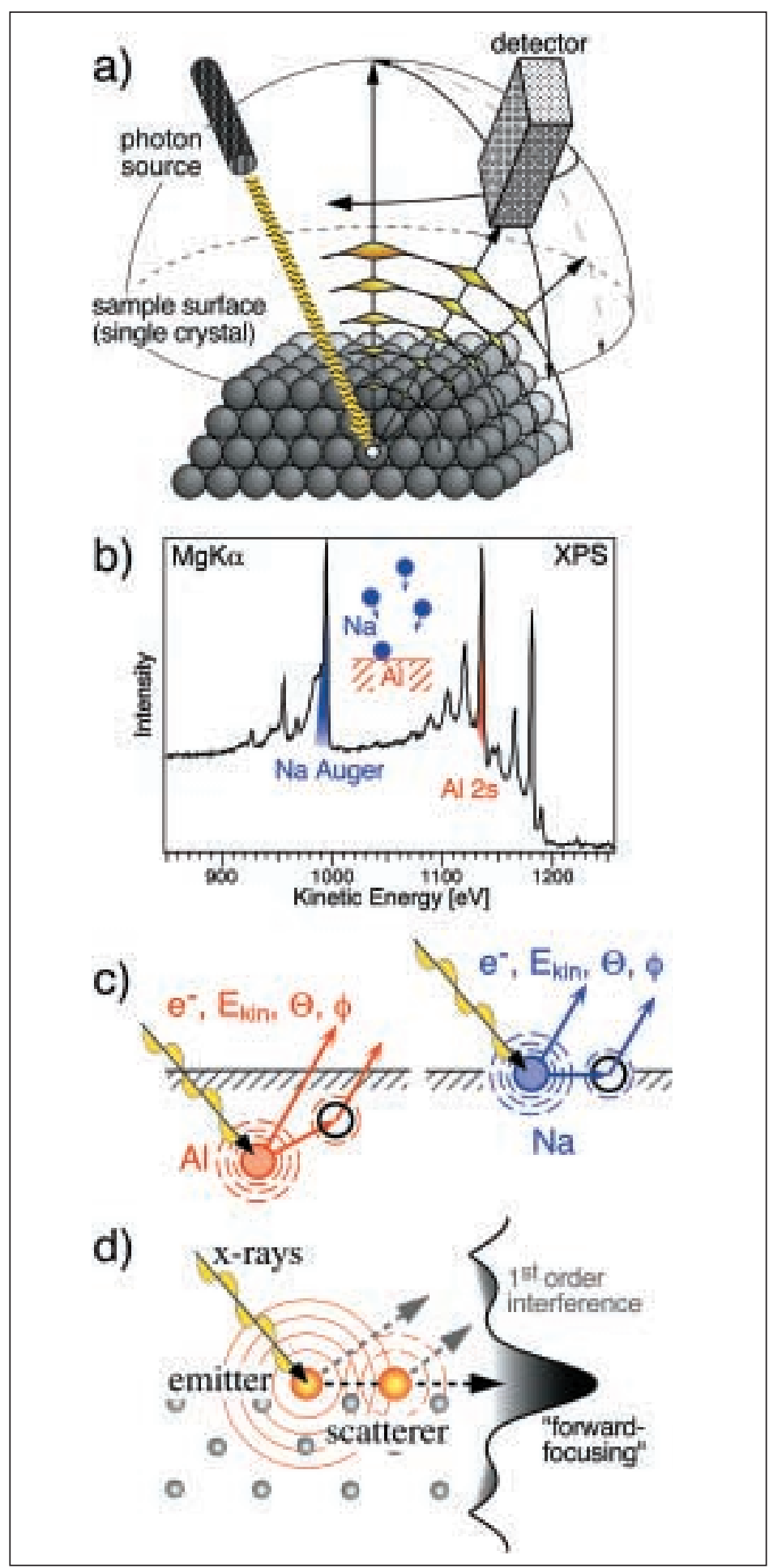

are present at the surface. $\mathrm{Na}$ atoms have been evaporated onto an $\mathrm{Al}(001)$ single crystal [2]. Since the photon is annihilated during the photoelectric effect giving its energy to the electron-hole formation, it is clear now that the different core levels can be distinguished when measuring the intensity of emitted photoelectrons as a function of kinetic energy in XPS. A core-hole can also decay by means of an Auger electron emission and, therefore, also Auger lines are present in XPS spectra. Collecting electrons from a particular emission line, e.g. the $\mathrm{Al} 2 \mathrm{~s}$ or the $\mathrm{Na}$ Auger line, we know that these electrons have their origin at the atom
Fig. 1. a) General principle of angle-scanned photoemission: Photoelectron intensities are collected over much of the hemisphere above the sample surface. b) X-ray photoemission spectrum for $\mathrm{Na}$ atoms deposited on $\mathrm{Al}(001)$ : Atoms have specific energy levels and, therefore, also photo- and Auger-emission lines at characteristic electron kinetic energies in the spectrum. c) By measuring the angular distribution (polar $(\Theta)$ and azimuthal $(\Phi)$ angle) of electron intensities from these characteristic lines the local real-space environment (geometry) around a chosen atom (emitter) can be monitored from the constructive or destructive interference. d) For sufficiently high electron kinetic energies $\left(E_{k i n}\right)$ the angular distribution can be interpreted in terms of 'forward focusing': most electrons are scattered in the forward direction (see text).

range of $0.5-3 \mathrm{~nm}$. Its value can be estimated from the universal curve for the electron mean free path [3].

As indicated in Fig. 1c, the experiment with X-ray excitation offers real-space information on the geometrical structure from the scattering of the photoelectron. Highly simplified, the photoabsorption process is sketched in Fig. 1d. As the photon is absorbed a photoelectron wave is moving out with an angular distribution depending on the polarization of the light and the initial state under consideration. The neighboring atoms act as scatterers. Relative vibrations infer damping from a Debye-Waller factor. For our purposes the outgoing wave is approximated by a plane wave at the location of the scatterer consisting of a spherically symmetric potential. Each scattered wave of angular momentum $l$ then undergoes a phase shift and the total scattering amplitude can be calculated as a function of scattering angle. Direct and scattered waves interfere constructively or destructively depending on angle, kinetic energy of the photoelectron and the geometrical arrangement around the emitter. Arriving at the surface the photoelectron is refracted due to the kinetic energy difference of the photoelectron inside and outside the solid. Furthermore the scattering process is limited by the elastic mean free path of the electron. In reality the scattering process is more complicated. Around each site the incoming spherical wave has to be fully developed into angular momentum components around the site before scattering and, in addition, multiple scattering has to be considered.

However, a much simpler approach is in the spectrum, e.g. an $\mathrm{Al}$ or a $\mathrm{Na}$ atom, respectively. As a matter of fact the intensity of the emission line in the spectrum not only changes due to the concentration of the species but also because of interference of the directly outgoing photoelectron wave with all scattered waves as a function of angle (Fig. 1c). Depending on the arrangement of the atoms near the emitting atom and the kinetic energy of the photoelectron, interference is constructive or destructive. The spatial extent of the arrangement thus probed is limited by the finite mean free path of the photoelectrons which is in the possible in many cases. If the photoelectron kinetic energy is chosen above approximately $0.5 \mathrm{keV}$ the scattering amplitude is mostly directed in the forward direction as shown in Fig. 1d. This so-called forward focusing leads to an almost geometrical interpretation where the emitted electrons simply produce a projection of near-neighbor directions, densely packed atomic rows and planes. Besides the so-called forwardfocusing intensity maxima, first order interference fringes allow the emitter-scatterer distance to be determined. 


\section{Results and Discussion}

\subsection{Surface Atom Positions and Crystallographic Structure}

In Fig. $2 a$ and $2 b$ the angular distribution of intensities of the $\mathrm{Na}$ and $\mathrm{Al}$ emission lines displayed in Fig. $1 \mathrm{~b}$ is shown. The patterns (gray-scale images, with high intensity in white) represent so-called diffractograms. Very strikingly the present example shows that such patterns may be very different for different atomic species reflecting their distinct atomic environment. The data is presented using a stereographic projection. The center of the gray-scale plots then corresponds to normal emission of electrons whereas the outer circle marks an emission angle of $\Theta=90^{\circ}$, i.e. emission along the surface plane. Data acquisition is performed sequentially such that for each polar emission angle, $\Theta$, the sample is rotated azimuthally ( $\Phi$ angle) around its normal. Thereby, the photon source and electron energy analyzer remain fixed in space.

It is straightforward now to interpret the diffractograms. We understand that the $\mathrm{Na}$ atoms are sitting at the very surface in the top layer with no atoms above. The emission from $\mathrm{Na}$ atoms does not induce any forward-focusing maxima at angles near normal emission, i.e. near the center of the diffractogram. On the contrary, high intensities are observed at the border for very grazing emission angles including circular segments representing the first order interference fringes.

For the $\mathrm{Al}$ emission the situation is completely different. Characteristic are the four intensity maxima at $45^{\circ}$ off normal (one of them is marked by a dot) and along the

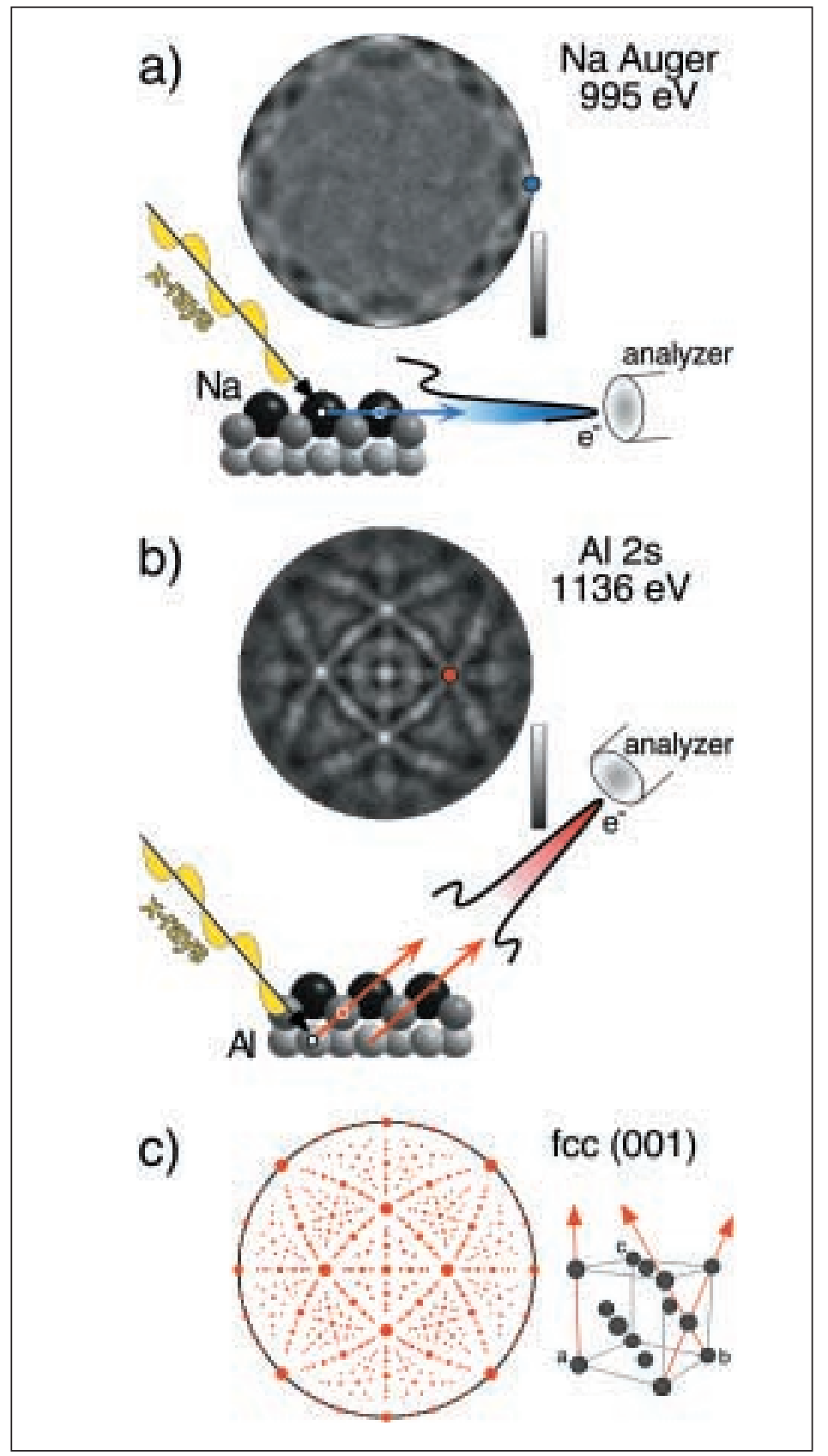

sample normal. They clearly correspond to high symmetry directions of the fcc crystal structure (Fig. 2c) of the Al substrate aligned with its normal along the (001) direction. The circular segments crossing through the four $45^{\circ}$ maxima correspond to densely packed crystallographic planes inherent to the fcc(001) structure. The circular lines appear due to the stereographic projection, which transforms planes into circles. Several substrate layers contribute to the signal resulting in a pattern fully consistent with the bulk crystallographic structure. Fig. 2c displays a projection of atomatom directions of the fcc(001) structure with the dot size inversely proportional to the emitter-scatterer distance. This arrangement of dots (Fig. 2c), obtained exclusively from geometry, achieves an excellent agreement with the experiment (Fig. 2b).

Due to the appearance of densely packed crystallographic planes in the diffraction pattern they can be taken as a fingerprint of the crystallographic structure near the surface even in the absence of long-range order, i.e. when no low-energy electron-diffraction pattern is detected [4]. It is obvious that from such XPD patterns it is straightforward to identify the growth orientation and structure, i.e. to distinguish between (001), (110), (111) orientation or between fcc, bcc or hcp structures, for ultra thin layers or clusters.

\subsection{Fingerprinting}

Another useful mode of XPD exists for host materials containing several different species and, in addition, that are doped with a particular element. Fig. 3 shows the XPD patterns measured on the $\mathrm{Bi}_{2} \mathrm{Sr}_{2} \mathrm{Ca}_{1} \mathrm{Cu}_{2} \mathrm{O}_{8+\delta}$ (Bi2212) high-temperature superconductor compound. It contains five different elements with diffractograms that are distinct. Often these materials are doped either to induce structural changes or to vary the critical temperature. Without studying in detail the possibly complicated crystallographic structure, the XPD pattern of the different host elements can be measured and considered as a characteristic image of the average local real-space environment of the particular element. In the present case Bi2212 has been doped with $\mathrm{Y}$ and $\mathrm{Pb}$, and XPD pat-

Fig. 2. Photoelectron diffraction experiments for a) the $\mathrm{Na}$ and b) the Al emission lines. Below each measurement a sketch illustrates the atomic structure based on the forward focusing interpretation. $\mathrm{Na}$ atoms sit on the surface, resulting in forward-focusing maxima and interference fringes only at grazing emission. Al emission is identical to bulk emission from clean $\mathrm{Al}(001)$ with 4 intensity maxima at $\Theta=45^{\circ}$ and circular lines of high intensity characteristic for the fcc structure of Al sketched in c). c) Stereographic projection of atom-atom directions for a cluster of atoms with fcc structure, weighted with the inverse emitter-scatter distance. Note the excellent agreement with the Al bulk signal from $b$ ). 
terns have been measured on corresponding emission lines. The pattern of the dopant may now easily be identified with one of the host element images resolving immediately the dopant site [5]. Comparing the top-most diffractograms it is perfectly clear that $\mathrm{Y}$ occupies $\mathrm{Ca}$ sites and $\mathrm{Pb} \mathrm{Bi}$ sites.

\subsection{Molecular Orientation}

Two of the structural key properties of adsorbed molecular films are the molecular orientation and the intramolecular rearrangement upon adsorption. For molecules larger than a few atoms, however, the determination of molecular orientation and conformation is by no means a trivial task. Most of the traditional structural methods fail in the case of too many atoms per unit cell, and spectroscopic methods probing transition matrix elements rely on the existence of a few but well-defined and separated symmetry elements. In the following, the potential of XPD for the structural investigation of molecular adsorbate system shall be outlined by a few exemplary cases. We will show that for adsorbed fullerene $\mathrm{C}_{60}$ molecules XPD provides rather direct structural information, allowing the determination of molecular orientation to a high degree of accuracy. We will see that de-

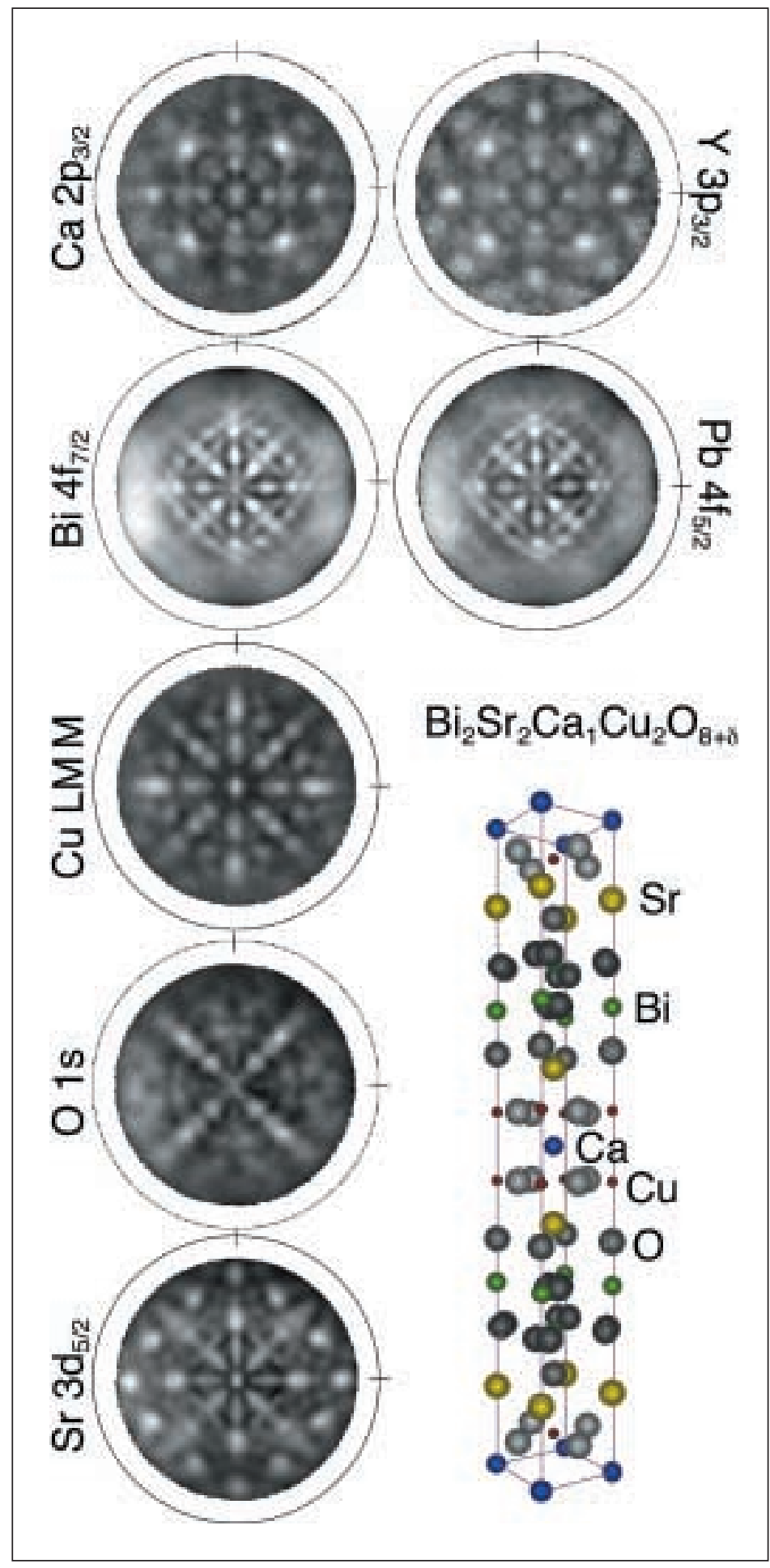

pending on the substrate surface and on surface coverage very different molecular orientations are observed. Results from $\mathrm{C}_{70}$ monomolecular films and calculations for adsorbed tartaric acid molecules shall demonstrate that the application of XPD is by no means limited to the case of $\mathrm{C}_{60}$ but that more complex molecular systems can successfully be investigated.

In recent years, the adsorption of $\mathrm{C}_{60}$ on metal surfaces has been extensively studied, and important changes in the structural, vibrational, and electronic properties of the fullerene molecules due to the interaction with the surface have been determined [6]. By now, it is well established that $\mathrm{C}_{60}$ forms a chemical bond with metal surface atoms, which, depending on the electronic properties of the surface, can be more or less ionic or covalent in character [7]. To optimize the bonding with the surface, the molecules can arrange in some preferred orientations, can become distorted, and, in some cases, induce structural instability and reconstruction of the substrate surface [6]. Therefore, an important property of these systems is the molecular orientation of the cage with respect to the surface, which is directly related to the interfacial interaction between the substrate and the molecules as well as to the nearest-neighbor molecule-molecule interaction.

The scattering situation for $\mathrm{C}_{60}$ molecule chemisorbed on a single-crystal surface is schematically shown in Fig. 4a. All the 60 carbon atoms of the molecule act as photoemitters, and the photoelectrons are scattered from the surrounding ion cores. Because of the forward focusing effect discussed above, intensity maxima are observed in directions corresponding to $\mathrm{C}-\mathrm{C}$ interatomic directions. At sufficiently high energies, the XPD pattern therefore is, to a first approximation, a forward-projected image of the atomic structure around each of the 60 carbon photoemitters. The XPD pattern thus represents a real-space 'fingerprint' of the particular molecular orientation. Furthermore, detailed structural parameters can be determined by comparing the experimental XPD patterns to calculated ones, optimizing the structural parameters until best agreement is achieved. The relatively simple and efficient single-scattering cluster (SSC) formalism [8] has proven adequate in most cases. Fig. $4 \mathrm{~b}$ shows an XPD pattern calculated for a $\mathrm{C}_{60}$ molecule facing with a six-membered ring

Fig. 3. XPD as a fingerprinting method: Diffraction patterns for the different species contained in the $\mathrm{Bi}_{2} \mathrm{Sr}_{2} \mathrm{Ca}_{1} \mathrm{Cu}_{2} \mathrm{O}_{8+\delta}$ high temperature superconductor together with patterns from dopant elements $\mathrm{Y}$ and $\mathrm{Pb}$. A simple comparison allows a straightforward identification of the $\mathrm{Y}$ and $\mathrm{Pb}$ sites. 
(6-ring) towards the surface, as sketched in Fig. 4a. In the right half of this plot, the interatomic directions within the molecule are indicated by black spots with sizes inversely proportional to the corresponding C-C distance. A correlation of dominant intensity maxima in the diffraction pattern and interatomic distances is clearly observed, and the diffraction pattern thus represents a real-space 'fingerprint' of the particular molecular orientation considered. This is also evidenced by the SSC calculation for a $\mathrm{C}_{60}$ molecule facing with a fivemembered ring (5-ring) towards the surface as shown in Fig. 4c, which in contrast to the three-fold symmetric calculation in Fig. 4b reflects the five-fold symmetry of a $\mathrm{C}_{60}$ molecule aligned with one of its five-fold symmetry axis along the surface normal.

Experimental $\mathrm{C}$ 1s diffraction patterns from monolayer $\mathrm{C}_{60}$ films on $\mathrm{Cu}(111)$,

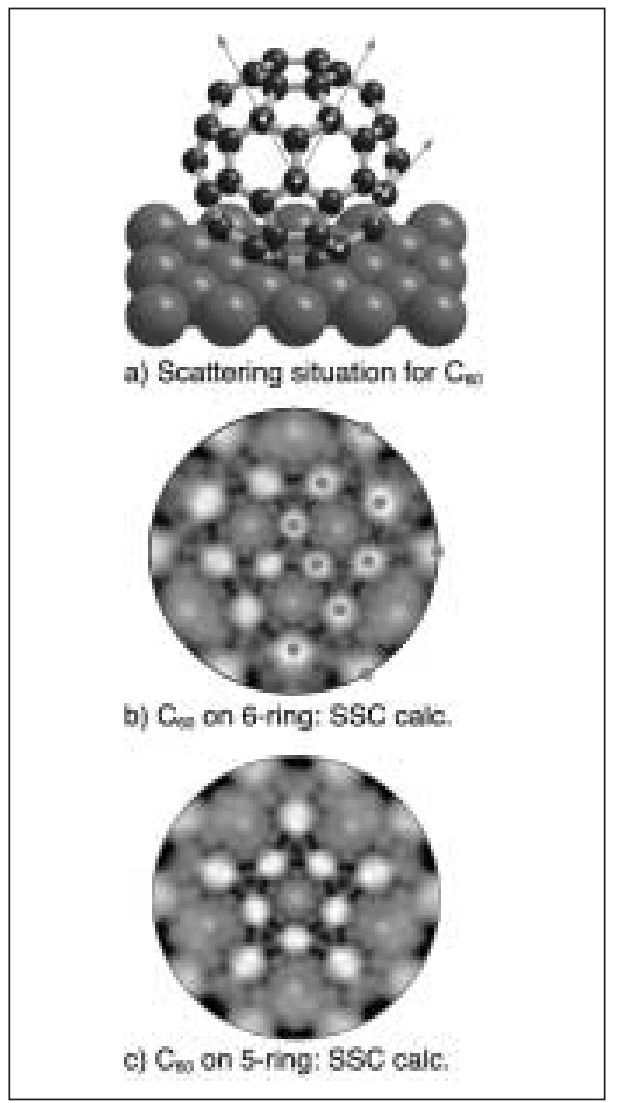

Fig. 4. XPD from chemisorbed $\mathrm{C}_{60}$ molecules. a) $C$ 1s photoelectrons are emitted from each of the 60 carbon atoms within the molecule and scattered from the surrounding ion cores. b) and c): Photoelectron diffraction patterns calculated for a $\mathrm{C}_{60}$ molecule facing with a 6ring (b) or a 5-ring (c) towards the surface. In the right half of $b$ ) the interatomic directions within the $\mathrm{C}_{60}$ molecule are indicated by dots with sizes inversely proportional to the corresponding $\mathrm{C}-\mathrm{C}$ distance. Correlation of dominant intensity maxima in the diffraction pattern and interatomic directions is observed, and the diffraction pattern thus represent a realspace 'fingerprint' of the particular molecular orientation considered.
$\mathrm{Cu}(110), \mathrm{Al}(001)$ and $\mathrm{Ni}(001)$ are shown in the left column of Fig. 5. By symmetry arguments alone, restrictions to the possible molecular orientations can immediately be made from these experimental data. For instance, the fivefold rotational symmetry of the $\mathrm{C}_{60}$ molecule facing with a 5-ring towards the surface excludes this orientation for all the systems presented in Fig. 5. It turns out that each of the diffraction patterns shown in Fig. 5 stems from a different molecular orientation, as shown in the right column of Fig. 5. On $\mathrm{Cu}(111)$, the $\mathrm{C}_{60}$ molecules are facing towards the surface with a 6-ring, on $\mathrm{Cu}(110)$ adsorption takes place on a $5-6$ bond aligned along the $<001\rangle$ surface directions, on $\mathrm{Al}(001)$ the $\mathrm{C}_{60}$ mole- cules are adsorbed facing with a single edge atom towards the substrate, whereas on $\mathrm{Ni}(001)$ the molecules bind to the surface through a 6-6 bond aligned along the $<100\rangle$ directions [9]. The SSC calculations for these configurations (middle column of Fig. 5) reproduce the experimental XPD patterns (left column of Fig. 5) very well not only the dominant diffraction features but also most of the fine structure is correctly reproduced. This is very promising in that also more complicated systems involving different inequivalent molecular orientations or some degree of orientational disorder might be successfully analyzed using XPD.

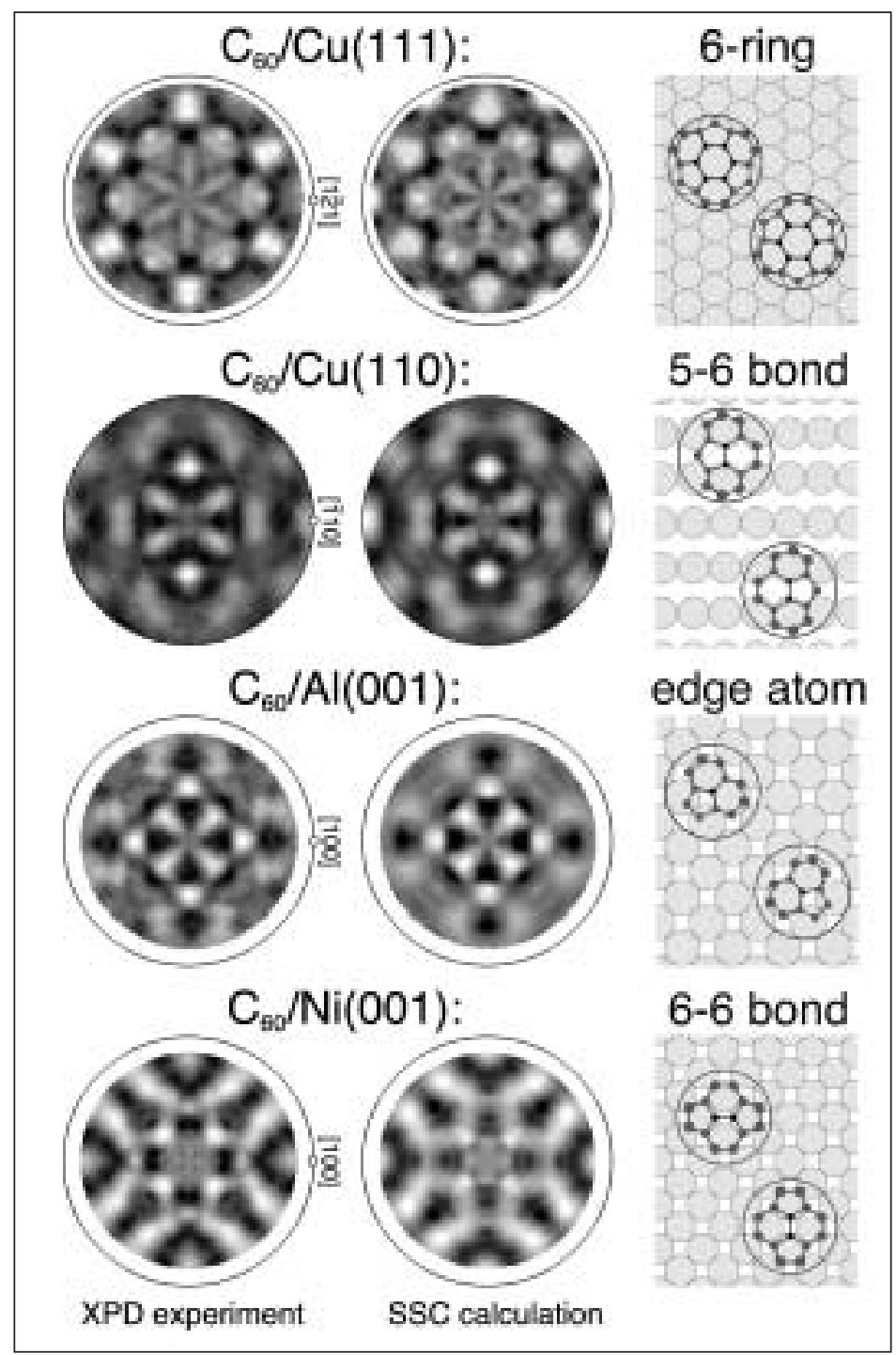

Fig. 5. Left column: Experimental $C$ 1s XPD patterns from monolayer $C_{60}$ films adsorbed on $\mathrm{Cu}(111), \mathrm{Cu}(110), \mathrm{Al}(001)$ and $\mathrm{Ni}(001)$. The orientation of the substrate surface as determined from substrate core-level XPD patterns (not shown) is indicated. Middle column: Best-fit single-scattering cluster calculations for the respective molecular orientations. The molecular orientations corresponding to these calculations are discussed in the text and schematically shown in the right column. Right column: Molecular orientations as determined from the XPD patterns. The substrates are aligned as indicated in the experimental XPD patterns. Substrate lattice spacings and $\mathrm{C}-\mathrm{C}$ distances are properly scaled. For clarity, only the lower carbon atoms of the molecules are shown. The atoms closest to the substrate surface are shown as black dots. The approximate size of the molecules is indicated. 
Motivated by the fact that $\mathrm{C}_{60}$ adsorption via a double bond bridging between the closed-packed rows of $\mathrm{Cu}(110)$ represents a rather asymmetric and unusual configuration, we have analyzed the $\mathrm{C}_{60} / \mathrm{Cu}(110)$ system in some more detail [10]. An extensive R-factor analysis comparing the experimental C 1s diffraction pattern to SSC calculations has been performed, allowing the $\mathrm{C}_{60}$ molecules to rotate away from their genuine 5-6 orientation. The best agreement is obtained if the 5-6 bond is rotated by $4.5^{\circ}$ away from the $\langle 001\rangle$ directions and the molecule is tilted by $4.5^{\circ}$ towards the bottommost 5-ring, as schematically shown in Fig. 6. These rotations away from the high-symmetry bonding configuration can be rationalized by the resulting better-balanced $\mathrm{C}-\mathrm{Cu}$ bond distance distribution and a maximization of the intermolecular $\mathrm{C}-\mathrm{C}$ distances from $2.9 \AA$ to $3.0 \AA$.

In the case of $\mathrm{C}_{60}$ adsorbed on the $\operatorname{Pd}(110)$ surface, deviations from symmetric adsorption configurations depend on $\mathrm{C}_{60}$ coverage and annealing temperature [11]. For low coverages and deposition at room temperature, the molecules are found to be tilted by a few degrees towards the bottommost 6-ring, whereas a deviation from high-symmetry is absent in regular structures formed at higher coverages after high-temperature annealing. Also intriguing is the case of the monolayer $\mathrm{C}_{60} / \mathrm{Ag}(001)$ system, where scanning tunneling microscopy images show an irregular distribution of two differently imaged molecules [12]. From the XPD data the presence of two inequivalent prevailing molecular orientations of the $\mathrm{C}_{60}$ cage has been determined, as well as a considerable fraction of rotationally disordered molecules. It is found that the two orientations

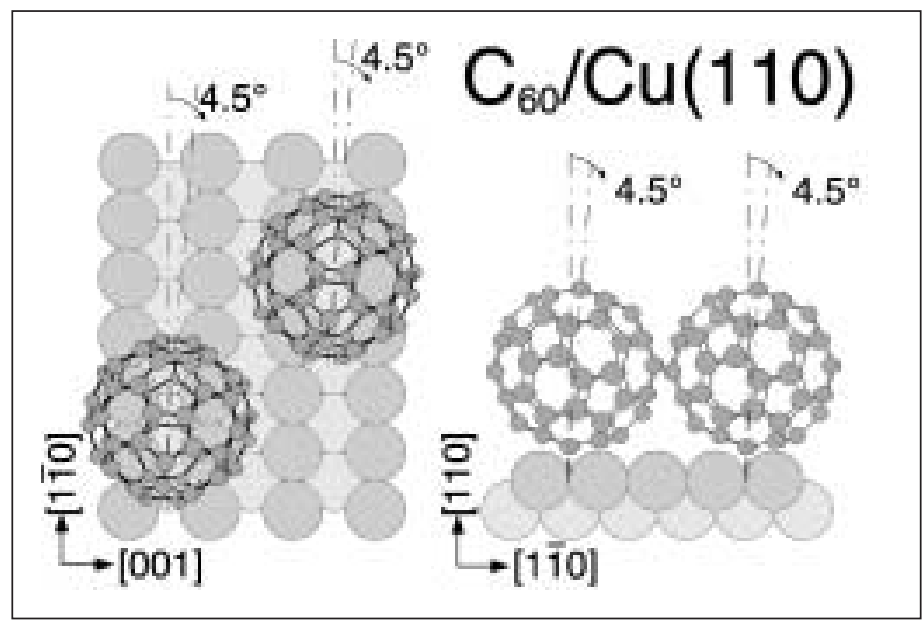

Fig. 6. Optimized molecular orientation of $\mathrm{C}_{60}$ adsorbed on $\mathrm{Cu}(110)$ : Top view (left) and side view (right). Arrows indicate rotations away from the symmetric 5-6 orientation. Nearest neighbor molecules are facing each other with six-membered rings, rotating the 5-6 bond closest to the surface by $4.5^{\circ}$ away from the [001] direction and inclining their vertical axis by $4.5^{\circ}$ towards the bottom five-membered ring. are not distinguished by the scanning tunneling microscope, and that the differently imaged molecules rather correspond to rotationally ordered and disordered molecular species [13].

These few examples have demonstrated that very detailed information about the molecular orientation of adsorbed $\mathrm{C}_{60}$ molecules can be gained from XPD data. That the same holds true also for less symmetrical molecules is shown in Fig. 7 where XPD data, SSC calculations and the optimized molecular orientations for the higher fullerene $\mathrm{C}_{70}$ adsorbed on $\mathrm{Al}(001)$ and $\mathrm{Cu}(110)$ surfaces are given. As in the case of $\mathrm{C}_{60}$, there is excellent agreement between experimental $\mathrm{C}$ 1s XPD patterns and best-fit SSC calculations. Interestingly, $\mathrm{C}_{70}$ adsorption on $\mathrm{Cu}(110)$ and $\mathrm{Al}(001)$ not only results in the same lateral arrangement of molecules (identical unit cells) as $\mathrm{C}_{60}$ adsorption on the respective surfaces, but $\mathrm{C}_{70}$ also binds with the same atom groups to the substrate as $\mathrm{C}_{60}$. On $\mathrm{Al}(001)$, both $\mathrm{C}_{70}$ and $\mathrm{C}_{60}$ face the substrate surface with a single edge atom. As for $\mathrm{C}_{60} / \mathrm{Cu}(110), \mathrm{C}_{70}$ adsorp-

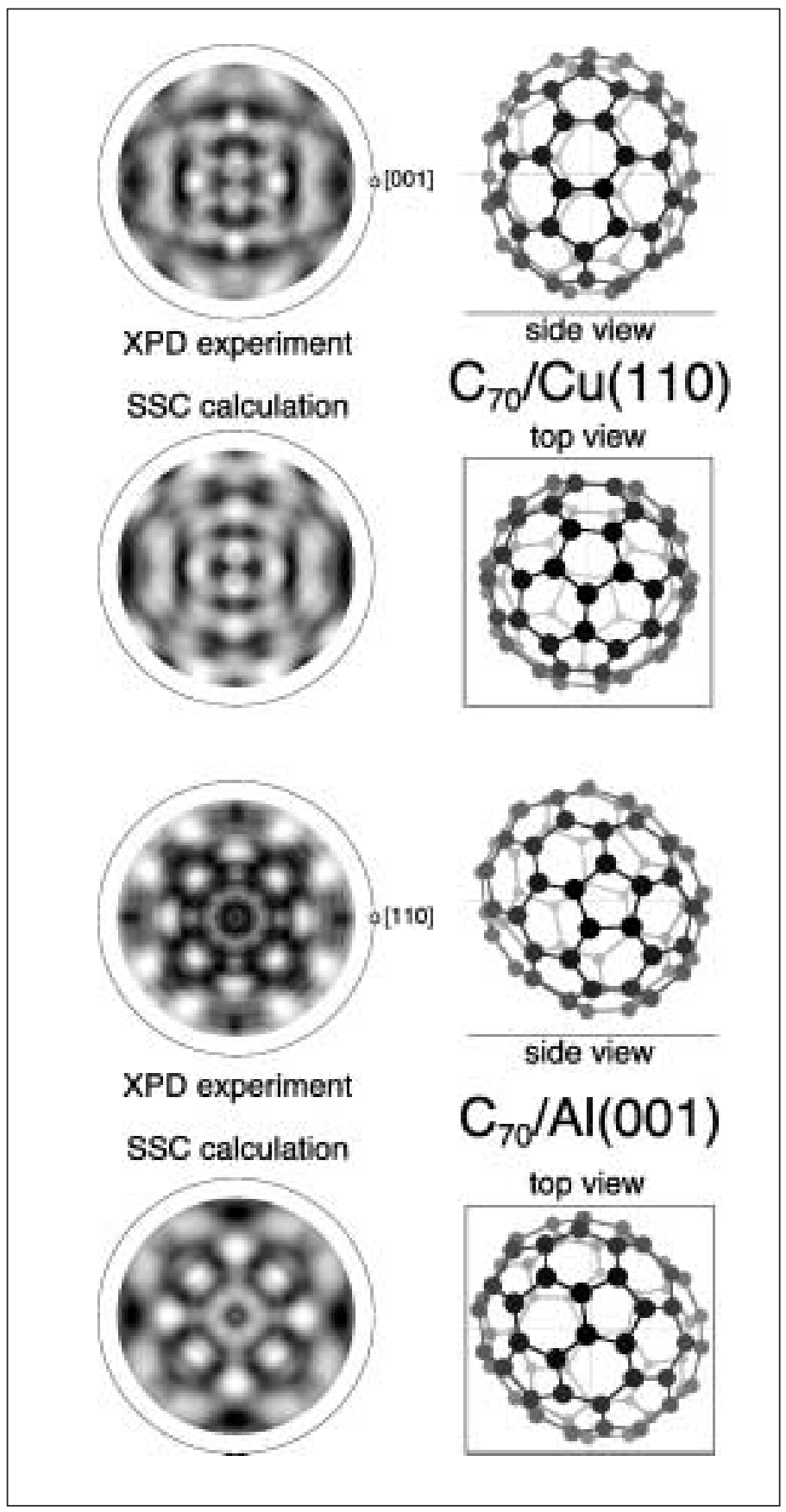

Fig. 7. Experimental C 1s XPD patterns, best-fit single-scattering calculations, and resulting $\mathrm{C}_{70}$ molecular orientations for monolayer $\mathrm{C}_{70}$ films adsorbed on $\mathrm{Cu}(110)$ and $\mathrm{Al}(001)$. The orientation of the substrate surface as determined from substrate core-level XPD patterns (not shown) is indicated in the C 1s XPD patterns. Interestingly, the $\mathrm{C}_{70}$ molecules are found to bind with the same atom group to the substrates than $\mathrm{C}_{60}$ on the respective surfaces (see text). 
tion on $\mathrm{Cu}(110)$ takes place on a 5-6 bond bridging between the closed-packed rows of surface atoms, with a small tilt of the molecule towards the bottommost 5-ring and a slight rotation of the 5-6 bond away from the <001> directions. Hence it seems that these atom groups provide optimum bonding with the respective substrate surface, and that the resulting binding energy dominates over the intermolecular $\mathrm{C}_{60}-\mathrm{C}_{60}$ or $\mathrm{C}_{70}-\mathrm{C}_{70}$ interactions.

The last two examples that shall be mentioned concern the adsorption of molecules that exhibit very few symmetry elements and exist in two enantiomeric forms. The orientation and intramolecular relaxation due to adsorption of the chiral phenanthrene-derivative heptahelicene, $\mathrm{C}_{30} \mathrm{H}_{18}$, on the $\mathrm{Cu}(111)$ surface has very recently been investigated by means of XPD [14]. Due to the low symmetry of the heptahelicene molecule the $\mathrm{C}$ 1s diffraction pattern from this system exhibits a scattering anisotropy as low as $2 \%$. Nevertheless, a detailed analysis involving simple molecular mechanics calculations of the atomic coordinates, photoelectron diffraction SSC calculations and an R-factor analysis permits the determination of the helicene molecular orientation and conformation. The molecules were found to bind to the substrate surface with their terminal phenanthrene group oriented parallel to the surface plane and to successively spiral away from the surface up to a height of $3.1 \AA$. This example nicely shows that not only the molecular orientation of a 'rigid' molecular cage but also conformational changes upon adsorption can be determined by XPD.

In this context, it is interesting to note that XPD has the potential to determine the handedness of an adsorbed chiral molecule, and to distinguish and identify the enantiomers, in a straightforward way. Consider the adsorption of tartaric acid (Fig. 8) on the $\mathrm{Cu}(110)$ surface, which Raval and coworkers have investigated in great detail [15]. For deposition temperatures above $350 \mathrm{~K}$ and low coverages, the tartaric acid molecules are found to be adsorbed solely in the bitartrate form, with the two carboxylate ends binding to the close-packed rows of the $\mathrm{Cu}(110)$ surface. $\mathrm{C}$ 1s SSC calculations for this bonding configuration are shown in Fig. 8 for the two enantiomers $(S, S)$ - and $(R, R)$-tartaric acid. As expected, the two diffraction patterns are mirror images of each other. Due to the fact that strong intensity maxima in the diffraction pattern correspond to emitter-scatterer directions and the emitters are known to be the carbon atoms, the position of the $\mathrm{OH}$-groups relative to the C-skeleton can immediately be determined from purely geometrical argu- ments. Therefore, a straightforward distinction and identification of enantiomers is possible in that case. Experiments along these lines are under way.

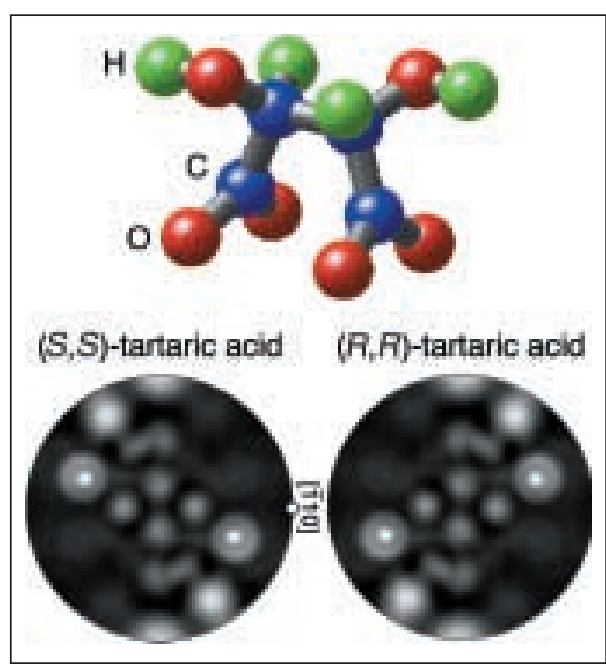

Fig. 8. C 1s photoelectron diffraction patterns calculated for the two enantiomers of tartaric acid adsorbed as bitartrates on $\mathrm{Cu}(110)$, with their two carboxylate ends binding to the close-packed rows of the $\mathrm{Cu}(110)$ surface. XPD clearly distinguishes the two enantiomers and allows their identification from purely geometrical arguments (see text).

\section{Conclusions and Outlook}

It has been shown that the chemical selectivity and sensitivity to the local atomic structure makes XPD a powerful method for the structural investigation of surfaces, to distinguish between different crystallographic surface structures, to identify impurity or dopant site positions and to unravel molecular orientation and conformation at surfaces. In particular, very detailed information about the molecular orientation of adsorbed fullerene molecules can be gained from XPD data. This detailed structural information calls for theoretical work: Theory is challenged to develop models that try to understand why the molecules behave the way they do and to explain their peculiar electronic properties.

All the results presented here have been collected with laboratory photon sources. With the increasing number of new, third generation synchrotron radiation sources the development of XPD is boosted. It is possible to achieve additional chemical sensitivity by using core-level shifted emission lines of identical atomic species that are in a different chemical environment such as in different layers or surrounded by different atoms. In addition, using sophisticated electron optics photoelectron microscopes are able to take XPD patterns from objects as small as $30 \mathrm{~nm}$.

\section{Acknowledgments}

Skillful technical assistance was provided by E. Mooser, O. Raetzo, R. Schmid, C. Neururer, and F. Bourqui. This Project has been supported by the Fonds National Suisse de la Recherche Scientifique.

Received: September 3, 2002

[1] J. Osterwalder, T. Greber, A. Stuck, L. Schlapbach, Phys. Rev. B 1991, 44, 13746; D. Naumović, A. Stuck, T. Greber, J. Osterwalder, L. Schlapbach, Phys. Rev. B 1993, 47, 7462.

[2] R. Fasel, P. Aebi. J. Osterwalder, L. Schlapbach, Surf. Sci. 1995, 331-333, 80; Phys. Rev. B 1995, 52, R2313; R. Fasel, P. Aebi, J. Osterwalder, L. Schlapbach, R.G. Agostino, G. Chiarello, Phys. Rev. B 1994, 50, 14516.

[3] M.P. Seah, W.A. Dench, Surf. Int. Anal. 1979, 1,2 .

[4] A. Fischer, R. Fasel, J. Osterwalder, A. Krozer, L. Schlapbach, Phys. Rev. Lett. 1993, 70, 1493.

[5] P. Schwaller, P. Aebi, H. Berger, C. Beeli, J. Osterwalder, L. Schlapbach, J. Electron Spectrosc. Relat. Phenom. 1995, 76, 127; T. Pillo, J. Hayoz, P. Schwaller, H. Berger, P. Aebi, L. Schlapbach, Appl. Phys. Lett. 1999, 75, 1550.

[6] For recent reviews, see P. Rudolf, in 'Proceedings of the Xth International Winterschool on Electronic Properties of Novel Materials. Fullerenes and Fullerene Nanostructures', Eds. H. Kuzmany, J. Fink, H. Hehring, and S. Roth, World Scientific, Singapore, 1996, pp. 263; A.V. Hamza, in 'Fullerenes: Chemistry, Physics and Technology', Eds. K.M. Kadish and R.S. Ruoff, Wiley, New York, 2000, pp. 531.

[7] A.J. Maxwell, P.A. Brühwiler, D. Arvanitis, J. Hasselström, M.K.-J. Johansson, N. Mårtensson, Phys. Rev. B 1998, 57, 7312.

[8] C.S. Fadley, in 'Synchrotron Radiation Research: Advances in Surface Science', Ed. R. Z. Bachrach, Plenum, New York, 1990, Vol. 1, pp. 421-518.

[9] R. Fasel, P. Aebi, R.G. Agostino, D. Naumović, J. Osterwalder, A. Santaniello, L. Schlapbach, Phys. Rev. Lett. 1996, 76, 4733; R. Fasel, PhD Thesis Nr. 1118, Université de Fribourg, 1996.

[10] R. Fasel, R.G. Agostino, P. Aebi, L. Schlapbach, Phys. Rev. B 1999, 60, 4517.

[11] J. Weckesser, C. Cepek, R. Fasel, J.V. Barth, F. Baumberger, T. Greber, K. Kern, J. Chem. Phys. 2001, 115, 9001.

[12] E. Giudice, E. Magnano, S. Rusponi, C. Boragno, U. Valbusa, Surf. Sci. 1998, 405, L561.

[13] C. Cepek, R. Fasel, M. Sancrotti, T. Greber, J. Osterwalder, Phys. Rev. B 2001, 63, 125406.

[14] R. Fasel, A. Cossy, K.-H. Ernst, F. Baumberger, T. Greber, J. Osterwalder, J. Chem. Phys. 2001, 115, 1020.

[15] M. Ortega Lorenzo, C.J. Baddeley, C. Muryn, R. Raval, Nature 2000, 404, 376; M. Ortega Lorenzo, S. Haq, T. Bertrams, P. Murray, R. Raval, C.J. Baddeley, J. Phys. Chem. B 1999, 103, 10661. 